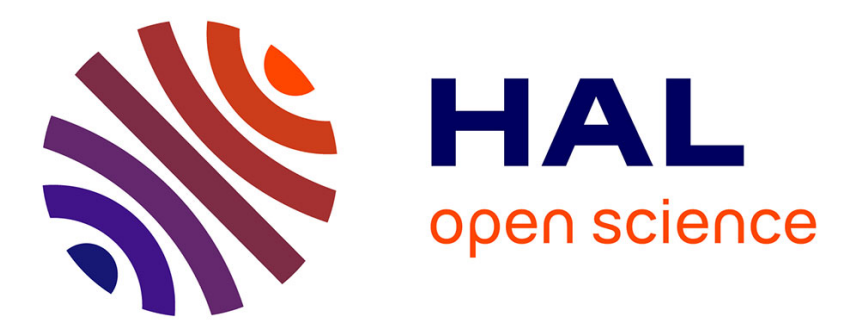

\title{
Multicellular scale front-to-rear polarity in collective migration
}

Lavinia Capuana, Astrid Boström, Sandrine Etienne-Manneville

\section{To cite this version:}

Lavinia Capuana, Astrid Boström, Sandrine Etienne-Manneville. Multicellular scale front-to-rear polarity in collective migration. Current Opinion in Cell Biology, 2019, 2020, pp.114 - 122. 10.1016/j.ceb.2019.10.001 . pasteur-02400670

\section{HAL Id: pasteur-02400670 \\ https://hal-pasteur.archives-ouvertes.fr/pasteur-02400670}

Submitted on 12 Dec 2019

HAL is a multi-disciplinary open access archive for the deposit and dissemination of scientific research documents, whether they are published or not. The documents may come from teaching and research institutions in France or abroad, or from public or private research centers.
L'archive ouverte pluridisciplinaire HAL, est destinée au dépôt et à la diffusion de documents scientifiques de niveau recherche, publiés ou non, émanant des établissements d'enseignement et de recherche français ou étrangers, des laboratoires publics ou privés. 


\section{Multicellular scale front-to-rear polarity in collective migration}

Lavinia Capuana $^{1,2}$, Astrid Boström ${ }^{1,3}$ and Sandrine Etienne-Manneville ${ }^{1}$

${ }^{1}$ Cell Polarity, Migration and Cancer Unit, Institut Pasteur, UMR3691 CNRS, Équipe Labellisée Ligue Contre le Cancer, F-75015, Paris, France.

${ }^{2}$ Sorbonne Université, Collège doctoral, F-75005 Paris, France.

${ }^{3}$ School of Life Sciences, College of Medical, Veterinary and Life Sciences, University of Glasgow, Glasgow G12 8QQ

Correspondence: setienne@pasteur.fr

Text : 3154 words

3 Figures

Declarations of interest: none

Abstract

Collective cell migration does not only reflect the migration of cells at a similar speed and in the same direction, it also implies the emergence of new properties observed at the level of the cell group. This collective behaviour relies on interactions between the cells and the establishment of a hierarchy amongst cells with leaders driving the group of followers. Here we make the parallel between the front-to-rear polarity axis in single cell and the front-to-rear multicellular scale polarity of a migrating collective which established through exchange of biochemical and mechanical information from the front to the rear and vice versa. Such multicellular scale polarity gives the migrating group the possibility to better sense and adapt to energy, biochemical and mechanical constrains and facilitate migration over long distances in complex and possibly changing environments. 
Collective cell migration is crucial for many physiological processes, from embryonic development to the adult where it participates for instance in wound healing and tissue renewal. Many pathologies have been linked with aberrant collective cell migration, cancer being a primary example [1-4]. Hence there is a need for a better understanding of the mechanisms controlling collective cell migration. During migration of single cells, the establishment of a front-to-rear polarity axis is crucial [5]. The protrusive front points to the direction of migration and is characterized by cytoskeleton-driven membrane protrusion. As the front protrudes, cell migration proceeds depending on the acto-myosin contractility which characterises the rest of the cell, including the cell rear. There, contractility also contributes to the detachment of cell adhesions to promote the translation of the cell. The formation of a front-to-rear axis is induced by polarity signalling which sees as major players the small GTPases proteins of the RHO family. Globally, at the front, CDC42 and RAC promote actin polymerisation and consequentially formation of protrusions such as filopodia or lamellipodia, whereas at the cell rear, Rho and other partners are controlling acto-contractility (For review see $[6,7])$. In the case of directed migration, biochemical and mechanical extracellular cues initially induce polarity signalling and the formation of a frontto-rear axis. When cells migrate in a persistent direction, several intracellular feedback loops involving for instance cytoskeletal organisation or membrane trafficking stabilise the front-to-rear polarity and migration direction.

Collective migration includes the migration of cell monolayers, cell groups of various size and shape, connected chains of cells, or more loosely connected cell streams. Model organisms such as Xenopus laevis, Drosophila melanogaster and Zebrafish, among others, have been crucial for the study and characterisation of collectively migrating cells. In parallel, in vitro approaches have been extensively used to elucidate key concepts underlying the establishment and maintenance of collective directed movements. Over the years, these analyses have shown that collective migration does not only characterise cells that move together in a similar direction but also imply that these cells migrate in a more directed manner and sometimes faster than cells migrating individually in similar conditions [6]. This collective behaviour emerges from cell cooperation within the group, allowed by intercellular communication through cell-cell contacts or soluble factors. Our better understanding of the relationships within migrating cell groups have led to the notion that front-to-rear polarity observed in single cell may be extended to a migrating cell group. Such multicellular-scale polarity relies on the establishment of a hierarchy amongst migrating cells. A population of front cells emerges from the migrating collective to serve as leaders which initiate, direct and facilitate the migration of followers, thanks to signalling molecules and transmission of forces through cell-cell contacts [6, 8, 9]. Similar to intracellular signalling observed in single polarised cells, information is exchanged within the cell collective either directly or indirectly in order to influence the behaviour of neighbouring cells, including the leaders, at both mechanical and biochemical levels [10]. As a result, migrating cell clusters, chains and sheets form highly polarised multicellular structures via mechanical and biochemical feedback loops flowing from the front to the back and vice-versa. This collective front-to-rear polarity provides a support not only for the most efficient migration, but also leads to the emergence of new properties such as collective chemotaxis, or durotaxis.

In this review, we first describe front-to-rear polarity within collectively migrating cell group. Subsequently, we focus on the latest advances showing how integration of polarity at a multicellular scale gives rise to the emerging properties of the cell collective, allowing them to sense and adapt to energical, biochemical and mechanical constrains. 
Cell-cell contacts tend to inhibit cell protrusion in a process called contact inhibition of locomotion (CIL), which plays a key role in the establishment of the front of migrating collectives $[6,11]$. Upon cell-cell interaction, membrane proteins including for instance cadherins, ephrins and PCP proteins, induce CIL through intracellular signalling and mechanical coupling. Cells located at the edge of the group harbour a free cell edge fundamentally distinct from their other sides engaged in cell-cell interactions. The asymmetric distribution of homophilic cadherin-mediated contacts is sufficient to promote cell polarisation towards the edge of the cell group [12]. Such mechanism restricts CDC42 and RAC activity at the contact free edge of these cells, leads to their polarisation towards the front of the migrating sheet and identify them as leader cells directing the migration of the entire monolayer [13] (Figure 1). Additional signalling can further restrict the number of leader cells. In epithelial sheet only a subpopulation of front cells become leaders and form finger-like structure at the wound edge [14]. Once the migration of leaders is initiated, the formation of contractile actin cables connecting them to the adjacent cells prevents RAC mediated protrusion in neighbouring cells $[15,16]$. The mechanical properties of the epithelial monolayer and the initial geometry of the front edge determine the distance between leader cells and thereby the size of the cell population following these leaders [16]. The size of the cell group following one leader depends on the length up to which forces can be transmitted [17].

The situation is more complicated in case of cell clusters where in principle all cells located at the edge of the cluster could become leaders. During border cell migration in the Drosophila ovary, a cluster of eight cells delaminate from the epithelium and migrate collectively between nurse cells towards the oocyte. The collective movement is ensured by the expression of DEcadherin which mediates contact between the border cells and between border cells and nurse cells. Rab11 acts on the whole cluster to promote the formation of a coherent actin-moesin structure that surrounds the cluster and restrict RAC-dependent protrusive activity in the leading cell $[18,19]$ (Figure 1). Rab11-mediated recycling of CDC42 controls the mechanical coupling within the cell cluster promoting mediated cytoskeletal tension through adherens junctions between adjacent cells [20]. As border cells migration proceeds, increased EGF signalling disrupts the acto-myosin cable surrounding the cell cluster and abolish the restriction of Rac activity to promote protrusion in all cells of the clusters which can then exchange position [21].

During mesenchymal migration of single cells, contraction between the protruding front and the retracting rear is mediated by ventral stress fibres that reach between focal adhesions located at the opposite sides of the cell (Figure 1). Cadherin-mediated contacts relay mechanical forces between the front and the rear of migrating groups (Figure 1). During migration of astrocyte or epithelial monolayer, most of the traction forces are exerted by the leaders with elevated RHOA activity, which pull on the followers [14, 22]. Mechanotransduction at cell-cell contacts and strengthening of adherens junctions at the front and rear of migrating cells can orient the actin cytoskeleton and mediate propagation of tension across the monolayer [9]. Vishwakarma et al. suggests a prominent role of forces generated by follower epithelial cells to induce and maintain leaders. Followers are pulling on the edge and by doing so they facilitate polarisation and protrusions formation in leaders [17]. P-cadherin mediated cell-cell contacts in myoblasts promote the concentration of CDC42 at the front edge of leaders and followers and homogenizes the cell polarity axis throughout the monolayer [23]. In migrating endothelial monolayers, VE-cadherinmediated adherens junctions at the rear of leader cell form protrusive fingers which penetrate the front of the following cells and triggers Rac-driven membrane protrusions [24]. The front-to-rear 
polarity of the leaders can thus propagate to followers and contribute to the global polarisation of migrating groups.

In addition to controlling multicellular scale cytoskeletal structures, many molecules involved in cell-cell interactions can recruit polarity complexes to the adhesion sites [11]. The polarised distribution of planar polarity proteins such as receptor protein tyrosine phosphatase Lar at the cell front and the cadherin Fat at the cell rear have recently been shown to sustain polarity signalling between neighbouring cells of the follicular epithelium in the Drosphila egg chamber $[25,26]$. In this model system, the planar polarisation of the semaphorin, Sema-5c also contributes to the global polarisation of the epithelium. At the leading edge of each cell, the activity of Lar is antagonized mechanism which involves the semaphorin, Sema-5c accumulates at the leading edge of each cell and signal through its receptor Plexin A to suppress protrusions in the cell ahead. It also antagonize Lar activity independently of Plexin A [27]. These recent observations illustrate how collective front-to-rear polarity is reinforced by multiple bi-directional polarity signalling at cell-cell interfaces (Figure 1).

\section{Collective polarization and energy sensing}

While in migrating monolayers of astrocytes or epithelial cells leaders are maintained at the front edge for a long period of time, in other systems exchanges between leaders and followers can occur. During migration of cranial neural crests (NCs) in Xenopus, chick and Zebrafish embryos, leader cells appear interchangeable. In this case, ablation of leaders does not totally bock migration, suggesting a rapid adaptation of followers to the formation of a free edge, generating an asymmetry in cadherin-mediated junctions [28].

During leukocyte single cell migration, the cell front samples the geometry of the migratory path and choose the path of least resistance, suggesting that the cell energy level is critical in migration [29]. Recent work from Reinhart-King`s group revealed the influence of cell energetic status in the dynamic polarity of migrating collective [30]. During breast cancer cell invasion, leader cells were shown to require more energy than followers, probably because they are responsible for a large part of the pushing and pulling forces required for migration through the extracellular matrix. Leader cells are thought to remodel the extracellular matrix and the formation of micro tracks for the less invasive follower cells [4, 31]. As collective migration progresses, the energy level of the leader cell decreases until it reaches a threshold level under which leaders cannot invade efficiently (Figure 2). Leader cells are then replaced by followers which become leaders. When put into a challenging environment, for example a denser collagen matrix, leaders' lifetime decreases, suggesting that the leaders-followers switch may be a collective strategy to sustain continued invasion [30]. Cancer cells may have a limited amount of energy to spend and thus exchange between leaders and followers occurs more rapidly. How the bioenergetic status of leaders controls the emergence of new leaders to overtake the failing leaders at the front remain unclear. Cadherin-mediated interaction may serve as sensors of the strength developed by leaders and thereby indicate to the followers when tractions forces are decreasing.

\section{Collective polarization and chemotaxis}

Extracellular biochemical cues that control single cell directed migration also contribute to collective migration $[15,16,32,33]$ (Figure 3A). During development, collective chemotaxis must occur over long distance during long period of time in a morphologically changing and growing 
embryo. In such context the maintenance of a steep enough chemokine gradient that could be sufficient to drive the cell group by influencing the direction and migration of each individual cell seems unlikely. Converging evidence indicate that the polarisation of the migrating cell groups facilitate long-distance chemotaxis (Figure 3A).

One simple mechanism is that leader cells are initially defined by a specific set of receptors which render them more susceptible to chemokine gradient. This is the case during border cells migration which is initiated by the secretion of specific signals produced by the oocytes (PVF (PDGF- and VEGF-related factor 1) and EGF [34]. The cell expressing the higher level of the receptor tyrosine kinase PVR shows a higher Rac activation and takes the role of leader driving the migration of the whole cluster $[1,35,36]$. Similarly a small population of cranial NCC present a specific transcriptomic signature which allows them to respond to a VEGF gradient and serve as leaders. In turn VEGF stimulation induces a change in gene expression reinforcing the leader cell phenotype [37]. Interestingly followers respond to ectopic expression of VEGF by adjusting their migratory path and their gene expression to become more "leader-like".

An emergent property of migrating collective is the self-generation of chemokine gradient, allowing the group to migrate in an initially homogenous medium. In the case of NCCs, computer modelling considering VEGF diffusion and uptake and assuming homogenous VEGF production showed that VEGF is progressively consumed by the cells at the front, creating a gradient of VEGF between the front and the rear of the cell group which is likely to improve the chemotactic behaviour [38]. The zebrafish lateral line primordium follows a path marked by a constant concentration of SDF1 (Stromal cell-derived factor 1) from the front to the rear of the lateral side of the animal. Front cells serving as leaders express the CXCR4, which upon SDF1 binding triggers migration. In contrast, followers situated in the second half of the primordium express a combination of CXCR4 and CXCR7. CXCR7 interacts with SDF1 without promoting migratory signals and sequesters the chemoattractant away from CXCR4, thereby generating a gradient of CXCR4 signalling between the front and the rear of the primordium [39, 40]. The self-generation of chemoattractant gradient is also used by melanoma cells [41, 42]. Melanoma cells placed in a medium containing LPA start to migrate after locally breaking down LPA using the lipid phosphatase 3 (LPP3) and creating a substantial outward-facing LPA gradient [41, 42]. This phenomenon is facilitated by high cell density, which increases LPA hydrolysis. This idea is in line with the fact that the "Breslow thickness", which gives an indication of the tumour cell density, is the most significant factor of poor prognosis for melanoma [43].

\section{Collective polarization and mechanosensing}

Both mechanical (substrate rigidity) and geometrical (confinement) characteristics of the cell environment influence the polarisation, the motility and the collective behaviour of migrating collectives.

Single cells can sense and follow gradient of ECM rigidity in a process called durotaxis (Figure 3B). Migrating collective can also undergo durotaxis, but the process appears much more efficient as it can be triggered in conditions where single cells are unable to durotax [44] . Making a parallel with what is observed during single cell durotaxis, it has been proposed that the control at the multicellular scale of the dynamics of cell-ECM adhesion via the focal adhesion molecular clutch, may explain the collective durotaxis [45] (Figure 3B). Transmission of acto-myosin forces across the cell monolayer via cell-cell junctions is essential [44], and may lead to an increased sensitivity to shallow rigidity gradient by integrating focal adhesion mechanical responses through a wider distance. While not analysed in this study, contribution of cell-cell contacts in the 
generation of cell polarity signalling is likely to play a key role. Although durotaxis in vivo has not been described, a beautiful example showing the importance of substrate stiffness in vivo, was described by Barriga et al. where they found that NCC in the Xenopus embryo undergo collective migration in response to a physiological change in the mesoderm stiffness underneath them. In this case only via establishing and maintaining cell-cell contacts, the cluster is able to keep directionality [46].

As single cell migration is influenced by the confinement which controls cell adhesion and spreading [47], force generation and speed of migration, the motile behaviour of confluent epithelial cells are influenced by confinement of the cell monolayer. When plated in domains of limited size epithelial monolayers exhibit collective pulsations as persistent random walkers adapt their motion to that of their neighbours. These results demonstrate that epithelial confinement alone can induce spontaneous collective motility and morphogenesis-like processes [48]. The amplitude and period of collective oscillatory motion of the confined epithelial sheets are dictated by the smallest confinement dimension [49]. At the single cell level the actin flow acts as a sensor of the cell edge curvature to influence migration at the single cell level [50]. It is tempting to speculate that cytoskeletal connection between cells of large cohorts may be used to sense mechanical information on a larger scale and adapt collective migration to the geometry of the environment. Accordingly cell-cell junctions transmit the forces and adapt polarity signalling to generate the collective behaviour observed in confined environment [49].

MDCK cells migrating in strips with a smaller width display an increased speed of migration, pointing to the hypothesis that a geometrical constraint would reduce the uncertainty of the leader cells and provide more directionality to the cluster [51]. Szabò et al. showed the importance of confinement during in vivo migration of NCCs in Xenopus embryos and identified the proteoglycan versican as confining molecule which acts as non-permissive substrate for the NCCs migration [52]. Chemotaxis alone cannot compensate for the lack of versican-induced confinement. More recently Semaphorin 3A was also shown to induce cephalic NC confinement by reducing cell adhesion to the extracellular matrix and Rac-dependent protrusive activity and thereby preventing SDF1-induced migration [53].

\section{Conclusions and perspectives}

Collective migration relies on the polarisation of the migrating cell group. Similarly to a single migrating cells, the front of a migrating collective points to the direction of migration and generate most of the forces necessary for the forward motion, while the rear is devoid of stable protrusive activity and instead often display a contractile periphery. Not only the polarity and the cytoskeletal organisation but also the energy level are coordinated at the multicellular. The collective behaviour appears to be mainly insured by cell-cell interactions and accumulating evidence point to the crucial role of direct cell-cell contacts and in particular of cadherin mediated adherens junctions. Cell-cell interactions insure the cohesion of migrating cell groups and they also contribute to the identification of leader cells at the front. Moreover they serve as mechanical bridges that transmit forces between the front and the rear of the cell collective and also as signalling relays which transduce polarity signalling. It is however likely that other structures involved in cell-cell communication, such as gap junctions, are involved. Biochemical and mechanical coordination between cells allows the cell group to sense chemokine gradient, rigidity gradient, geometry of the environment at a multicellular scale. In complex in vivo environment, the interplay between contact inhibition of locomotion (CIL) and chemotaxis, or chemotaxis and ECM-cell interactions may also trigger more efficient collective migration. For instance, in human 
hepatocarcinoma cells, cell-cell junctions trigger an increased level of chemokine receptors at the plasma membrane which promotes migration [54]. Furthermore, mathematical simulations have suggested that several chemoattractants must act together to define complex and long trajectories. For instance, three chemoattractants are needed to position the trunk NCC into the SG and the DRG [55]. Whether migrating collectives are better at integrating the effects of a large number of factors will need to be investigated.

\section{Aknowlegments}

This work was supported by La Ligue contre le cancer, the European Union Horizon 2020 Marie Skłodowska-Curie research and innovation programme (MSCA-ITN-2015-675407) and the Institut Pasteur. LC was funded by the Fondation ARC pour la recherche sur le cancer. We apologize to authors whose work we were unable to discuss due to space constraints.

\section{References}

1. Rorth, P., Collective cell migration. Annu Rev Cell Dev Biol, 2009. 25: p. 407-29.

2. Te Boekhorst, V., L. Preziosi, and P. Friedl, Plasticity of Cell Migration In Vivo and In Silico. Annu Rev Cell Dev Biol, 2016. 32: p. 491-526.

3. Friedl, P., et al., Classifying collective cancer cell invasion. Nat Cell Biol, 2012. 14(8): p. 77783.

4. Friedl, P., et al., New dimensions in cell migration. Nat Rev Mol Cell Biol, 2012. 13(11): p. 743-7.

5. Llense, F. and S. Etienne-Manneville, Front-to-rear polarity in migrating cells, in Cell polarity K. Ebnet, Editor. 2015, Springer. p. 115-146.

6. Mayor, R. and S. Etienne-Manneville, The front and rear of collective cell migration. Nat Rev Mol Cell Biol, 2016. 17(2): p. 97-109.

7. Pollard, T.D. and J.A. Cooper, Actin, a central player in cell shape and movement. Science, 2009. 326(5957): p. 1208-12.

8. Trepat, X., Z. Chen, and K. Jacobson, Cell migration. Compr Physiol, 2012. 2(4): p. 2369-92.

9. De Pascalis, C. and S. Etienne-Manneville, Single and collective cell migration: the mechanics of adhesions. Mol Biol Cell, 2017. 28(14): p. 1833-1846.

10. Friedl, P. and R. Mayor, Tuning Collective Cell Migration by Cell-Cell Junction Regulation. Cold Spring Harb Perspect Biol, 2017. 9(4).

11. Ebnet, K., et al., Regulation of cell polarity by cell adhesion receptors. Semin Cell Dev Biol, 2018. 81: p. 2-12.

12. Dupin, I., E. Camand, and S. Etienne-Manneville, Classical cadherins control nucleus and centrosome position and cell polarity. J Cell Biol, 2009. 185(5): p. 779-86.

13. Camand, E., et al., $\mathrm{N}$-cadherin expression level modulates integrin-mediated polarity and strongly impacts on the speed and directionality of glial cell migration. J Cell Sci, 2012. 125(Pt 4): p. 844-57.

14. Reffay, M., et al., Interplay of RhoA and mechanical forces in collective cell migration driven by leader cells. Nat Cell Biol, 2014. 16(3): p. 217-23.

15. Riahi, R., et al., Notch1-DI/4 signalling and mechanical force regulate leader cell formation during collective cell migration. Nat Commun, 2015. 6: p. 6556. 
16. Kollimada, S.A., et al., Advancing Edge Speeds of Epithelial Monolayers Depend on Their Initial Confining Geometry. PLoS One, 2016. 11(4): p. e0153471.

17. *Vishwakarma, M., et al., Mechanical interactions among followers determine the emergence of leaders in migrating epithelial cell collectives. Nat Commun, 2018. 9(1): p. 3469.

This study analyses the emergence of leader cells at the epithelial wound margin. The authors propose a non-cell autonomous mechanism where as a results of forces imbalance in the monolayer, followers pull on future leader cells facilitating their polarisation. Once established, the forces exerted by the leader prevent the emergence of other leaders.

18. Ramel, D., et al., Rab11 regulates cell-cell communication during collective cell movements. Nat Cell Biol, 2013. 15(3): p. 317-24.

19. Cai, D., et al., Mechanical feedback through E-cadherin promotes direction sensing during collective cell migration. Cell, 2014. 157(5): p. 1146-59.

20. Colombie, N., et al., Non-autonomous role of $\mathrm{Cdc42}$ in cell-cell communication during collective migration. Dev Biol, 2017. 423(1): p. 12-18.

21. Combedazou, A., et al., Small GTPases orchestrate cell-cell communication during collective cell movement. Small GTPases, 2017: p. 1-10.

22. De Pascalis, C., et al., Intermediate filaments control collective migration by restricting traction forces and sustaining cell-cell contacts. J Cell Biol, 2018. 217(9): p. 3031-3044.

23. Plutoni, C., et al., P-cadherin promotes collective cell migration via a Cdc42-mediated increase in mechanical forces. J Cell Biol, 2016. 212(2): p. 199-217.

24. Hayer, A., et al., Engulfed cadherin fingers are polarized junctional structures between collectively migrating endothelial cells. Nat Cell Biol, 2016. 18(12): p. 1311-1323.

25. *Barlan, K., M. Cetera, and S. Horne-Badovinac, Fat2 and Lar Define a Basally Localized Planar Signaling System Controlling Collective Cell Migration. Dev Cell, 2017. 40(5): p. 467477 e5.

This paper describes a novel mechanism of planar signalling promoting epithelial collective migration. Fat2 expression in one cell inhibits the formation of protrusion in the following cell by stabilising Lar at its leading edge.

26. Horne-Badovinac, S., Fat-like cadherins in cell migration-leading from both the front and the back. Curr Opin Cell Biol, 2017. 48: p. 26-32.

27. ** Stedden, C.G., et al., Planar-Polarized Semaphorin-5c and Plexin A Promote the Collective Migration of Epithelial Cells in Drosophila. Curr Biol, 2019. 29(6): p. 908-920 e6.

In this manuscript, the authors show that the planar polarization of the transmembrane protein Sema-5c and its receptor Plexin A reinforces the front-to-rear polarization of the actin-based protrusion and collective behavior of follicular epithelial cells in Drosophila.

28. Richardson, J., et al., Leader Cells Define Directionality of Trunk, but Not Cranial, Neural Crest Cell Migration. Cell Rep, 2016. 15(9): p. 2076-88.

29. ** Renkawitz, J., et al., Nuclear positioning facilitates amoeboid migration along the path of least resistance. Nature, 2019. 568(7753): p. 546-550.

Leukocytes are highly motile amoeboid cells. They are characterised by scarce ability to degrade the matrix they are moving in, in contrast with mesenchymal cells. Instead, by positioning their nuclei in the cell front, when energy levels are limiting, leukocytes can discriminate the pore size, choose the larger paths and limit energy consumption. 
30. $* *$ Zhang, J., et al., Energetic regulation of coordinated leader-follower dynamics during collective invasion of breast cancer cells. Proc Natl Acad Sci U S A, 2019. 116(16): p. 78677872.

This article shows that cancer cells migrate collectively and by doing so acquire an increased invasive and migratory capacities. Leaders and followers are characterised by different energetic status. Cells with higher intracellular energy emerge as leaders until forward invasion consumes most of the available energy. Leaders are then replaced by followers displaying a higher energy level.

31. Cheung, K.J. and A.J. Ewald, A collective route to metastasis: Seeding by tumor cell clusters. Science, 2016. 352(6282): p. 167-9.

32. Theveneau, E. and C. Linker, Leaders in collective migration: are front cells really endowed with a particular set of skills? F1000Res, 2017. 6: p. 1899.

33. Caley, M.P., V.L. Martins, and E.A. O'Toole, Metalloproteinases and Wound Healing. Adv Wound Care (New Rochelle), 2015. 4(4): p. 225-234.

34. Prasad, M., et al., Border Cell Migration: A Model System for Live Imaging and Genetic Analysis of Collective Cell Movement. Methods Mol Biol, 2015. 1328: p. 89-97.

35. Bianco, A., et al., Two distinct modes of guidance signalling during collective migration of border cells. Nature, 2007. 448(7151): p. 362-5.

36. Inaki, M., et al., Effective guidance of collective migration based on differences in cell states. Proc Natl Acad Sci U S A, 2012. 109(6): p. 2027-32.

37. McLennan, R., et al., Neural crest migration is driven by a few trailblazer cells with a unique molecular signature narrowly confined to the invasive front. Development, 2015. 142(11): p. 2014-25.

38. McLennan, R., et al., Multiscale mechanisms of cell migration during development: theory and experiment. Development, 2012. 139(16): p. 2935-44.

39. Dona, E., et al., Directional tissue migration through a self-generated chemokine gradient. Nature, 2013. 503(7475): p. 285-9.

40. Dalle Nogare, D., et al., Leading and trailing cells cooperate in collective migration of the zebrafish posterior lateral line primordium. Development, 2014. 141(16): p. 3188-96.

41. Muinonen-Martin, A.J., et al., Melanoma cells break down LPA to establish local gradients that drive chemotactic dispersal. PLoS Biol, 2014. 12(10): p. e1001966.

42. * Susanto, O., et al., LPP3 mediates self-generation of chemotactic LPA gradients by melanoma cells. J Cell Sci, 2017. 130(20): p. 3455-3466.

Melanoma cells can initiate their own migration by generating lysophosphatidic acid (LPA) gradient. LPA is highly concentrated around the tumour and cells have hydrolyse it to then migrate through this self-generated gradient. The lipid phosphatase LPP3 is particularly important for LPA break down and therefore in the control of tumour invasion.

43. Gershenwald, J.E. and R.A. Scolyer, Correction to: Melanoma Staging: American Joint Committee on Cancer (AJCC) 8th Edition and Beyond. Ann Surg Oncol, 2018. 25(Suppl 3): p. 993-994.

44. Sunyer, R., et al., Collective cell durotaxis emerges from long-range intercellular force transmission. Science, 2016. 353(6304): p. 1157-61.

45. Elosegui-Artola, A., X. Trepat, and P. Roca-Cusachs, Control of Mechanotransduction by Molecular Clutch Dynamics. Trends Cell Biol, 2018. 28(5): p. 356-367.

46. * Barriga, E.H., et al., Tissue stiffening coordinates morphogenesis by triggering collective cell migration in vivo. Nature, 2018. 554(7693): p. 523-527. 
This study focuses on tissue mechanics and its importance during collective cell migration in vivo. By looking at the Xenopous laevis neural crest cells migration, they found that during morphogenesis, the tissue underneath the cephalic neural crest cells stiffens. This change in the mechanical characteristic of the tissue, leads to the initiation of an epithelial to mesenchymal transition in the neural crest and induces their migration.

47. Mohammed, D., et al., Substrate area confinement is a key determinant of cell velocity in collective migration. Nature Physics, 2019. 15(8): p. 858-866.

48. Deforet, M., et al., Emergence of collective modes and tri-dimensional structures from epithelial confinement. Nat Commun, 2014. 5: p. 3747.

49. * Peyret, G., et al., Sustained Oscillations of Epithelial Cell Sheets. Biophys J, 2019.

The authors show that epithelial cell sheets are highly plastic which allow them to undergo large deformation during tissue formation. They demonstrate that confinement induces collective oscillations involving cell-cell junctions, and forces transmission.

50. Chen, T., et al., Large-scale curvature sensing by directional actin flow drives cellular migration mode switching. Nat Phys, 2019. 15: p. 393-402.

51. Vedula, S.R., et al., Emerging modes of collective cell migration induced by geometrical constraints. Proc Natl Acad Sci U S A, 2012. 109(32): p. 12974-9.

52. Szabo, A., et al., In vivo confinement promotes collective migration of neural crest cells. J Cell Biol, 2016. 213(5): p. 543-55.

53. Bajanca, F., et al., In vivo topology converts competition for cell-matrix adhesion into directional migration. Nat Commun, 2019. 10(1): p. 1518.

54. Cepeda, E.B., et al., Mechanisms regulating cell membrane localization of the chemokine receptor CXCR4 in human hepatocarcinoma cells. Biochim Biophys Acta, 2015. 1853(5): p. 1205-18.

55. Dyson, L., et al., A chemotactic model of trunk neural crest cell migration. Genesis, 2018. 56(9): p. e23239. 
Figure 1: Front-to-rear polarity in single and collectively migrating cells. This figure illustrates that a parallel can be drawn between the front-to-rear polarity of single migrating cells in which the leading edge is characterized by a higher RAC/CDC42 activity at the front and the inhibition of Racmediated protrusion on the lateral and rear sides of the cell $(A)$ and what is observed in migrating collectives such as epithelial monolayers (B) or Drosophila border cells (C). In case of collective migration, cell-cell contacts between cells organize the actin cytoskeleton to transmit forces from the front to the rear and limit lateral or rear protrusions. They also contribute to feedback mechanisms improving front-to-rear polarity of the cell group.

Figure 2: Energy levels affect front-to-rear polarity in collectively migrating cells.

During collective migration in a 3D complex environment, leader cells use more energy than the followers to create the migration path and facilitate the migration of followers. When the energy level of the leaders becomes too low to achieve these tasks, a follower with a higher energy level takes over. The overall front-to-rear polarity and the high speed of migration can thus be maintained.

Figure 3: Similarities between single cell and collective chemotaxis and durotaxis.

Cell groups like single cells can polarize in response to biochemical (chemotaxis, A) and rigidity (durotaxis, B) gradients present in the microenvironment. Large cell groups are able to sense and respond to more shallow gradients. Moreover, once polarized cell groups can generate their own chemical gradient, as well as an mechanical easier migratory path. The multi-cellular scale polarization thus allows migrating collective to undergo a more directed migration. 


\section{A. Single migrating cell (typical fibroblast cell)}

MTOC/centrosome

\section{$\int$ Microtubules}

Focal adhesion

$\Longrightarrow$ Actin meshwork

$\longrightarrow$ Actin arcs

Acto-myosin cables

- Adherens junctions

Feedback stabilising polarity signalling

Forces transmission

between front cell to its follower
Rear

Front

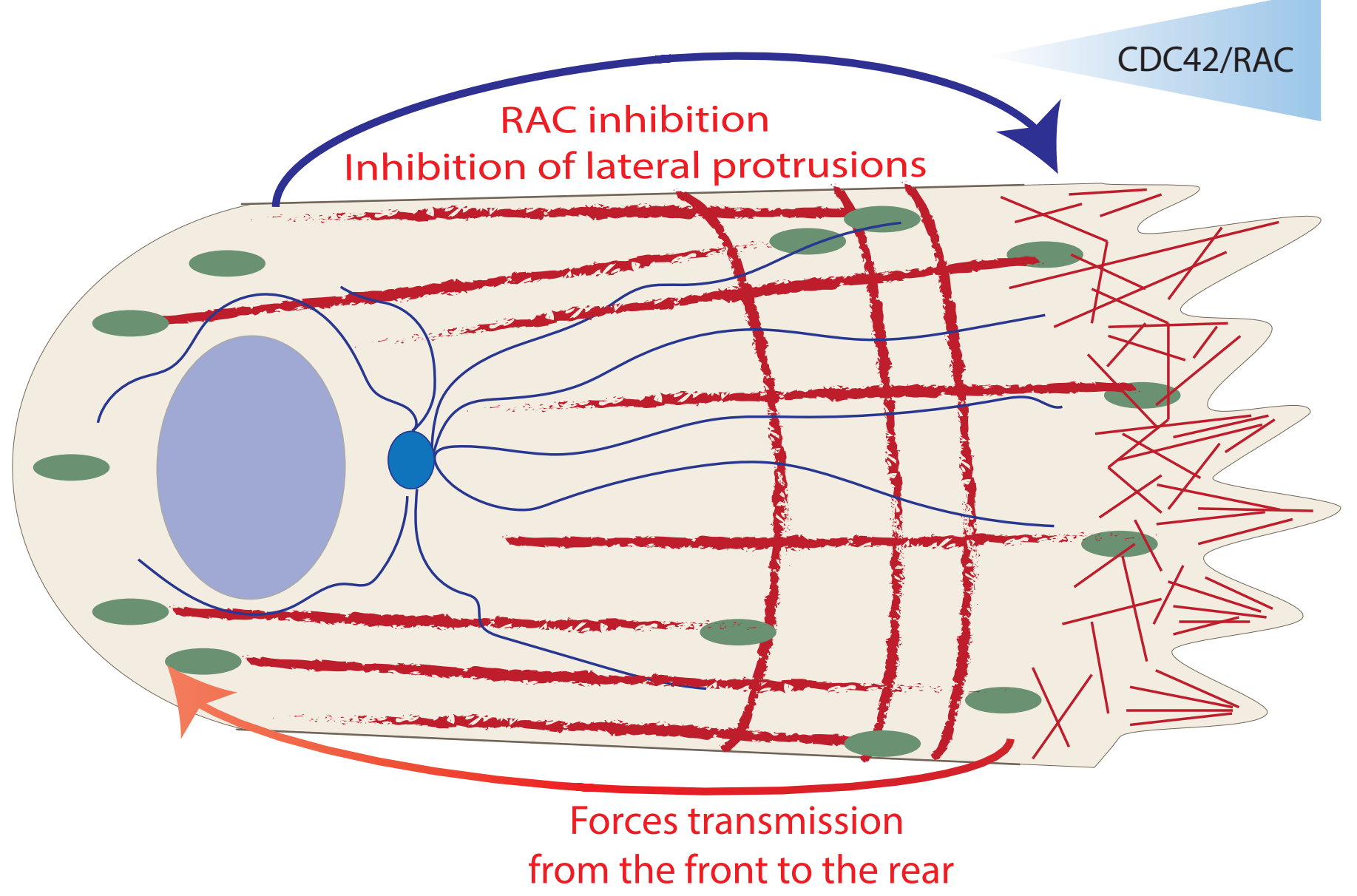

\section{B. Collective migration of a cell sheet (epithelial cell migration)}

Rear Front

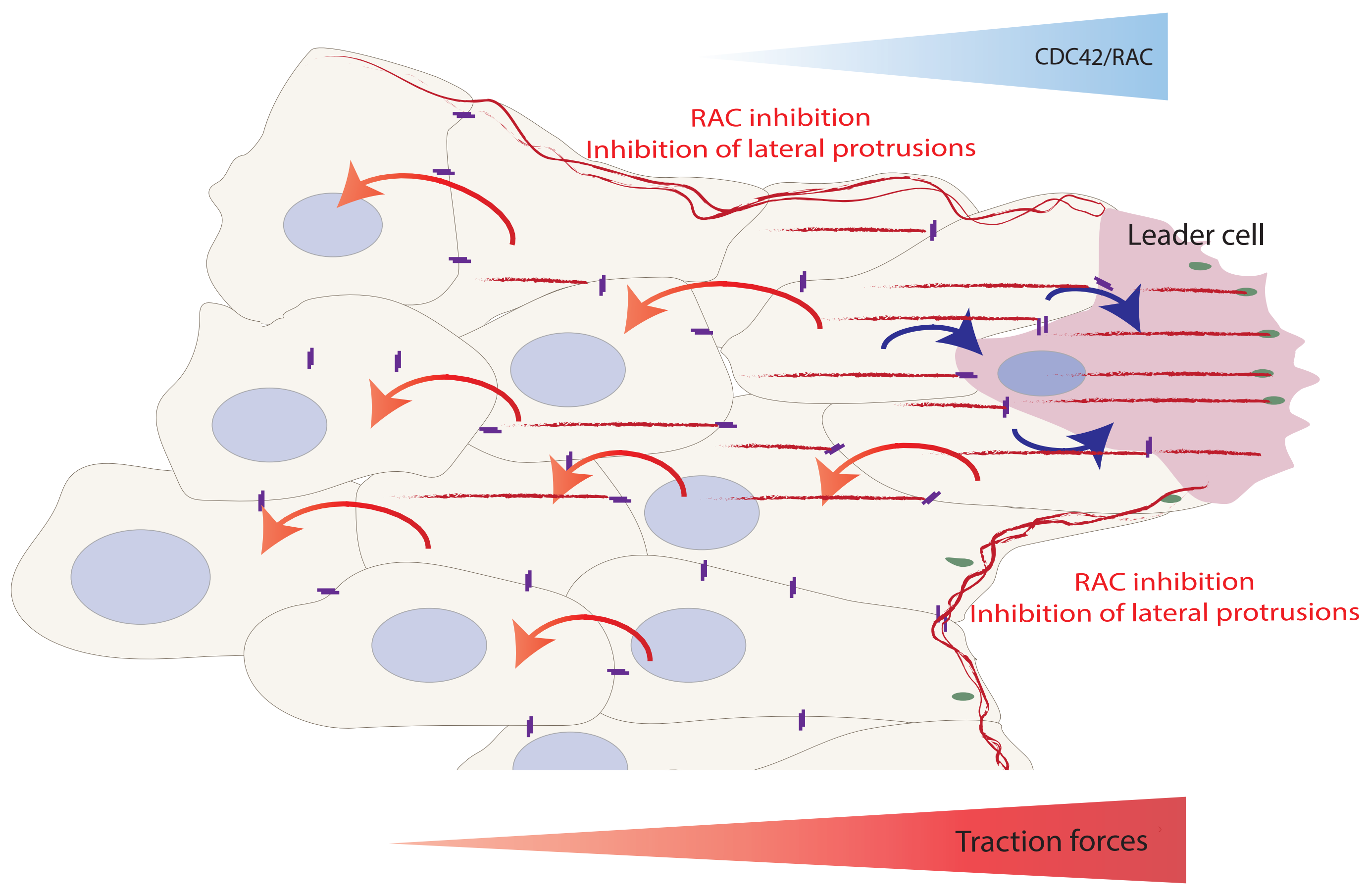

C. Collective migration of a Drosophila border cells

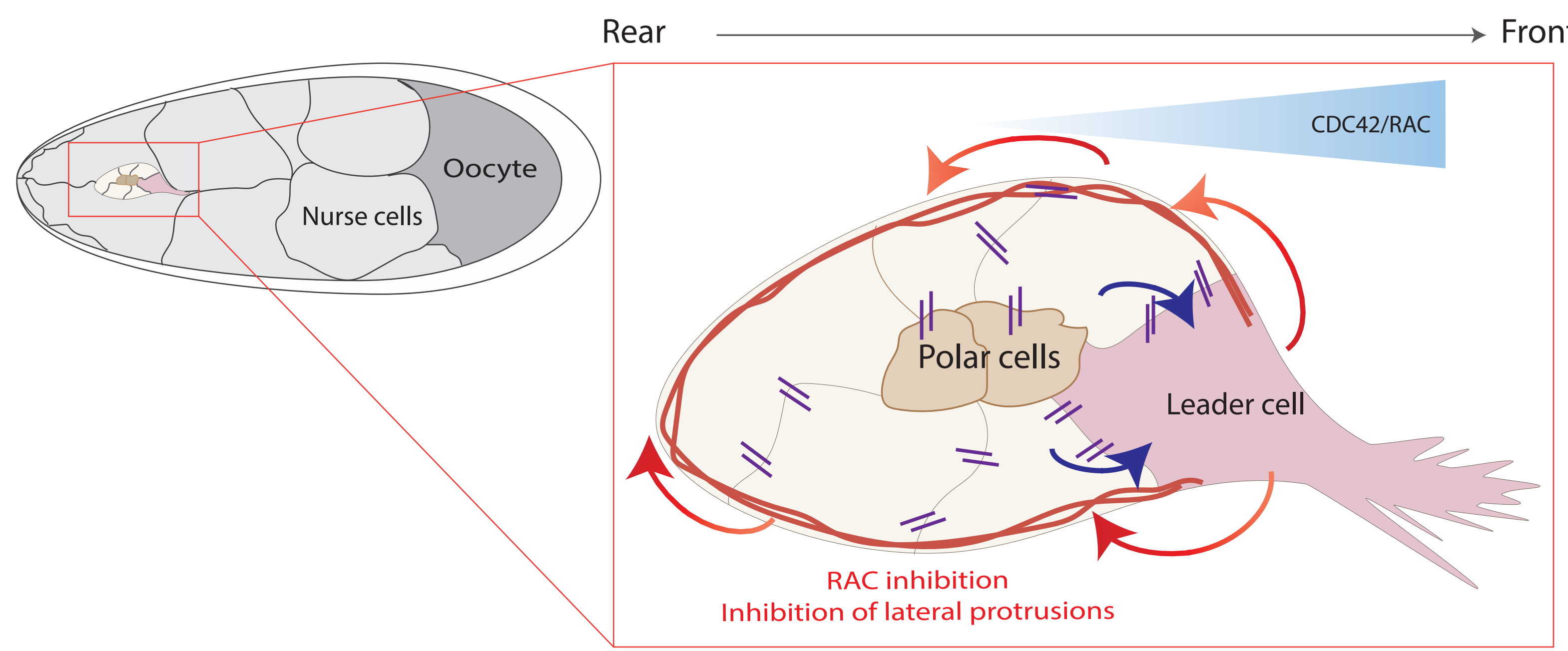




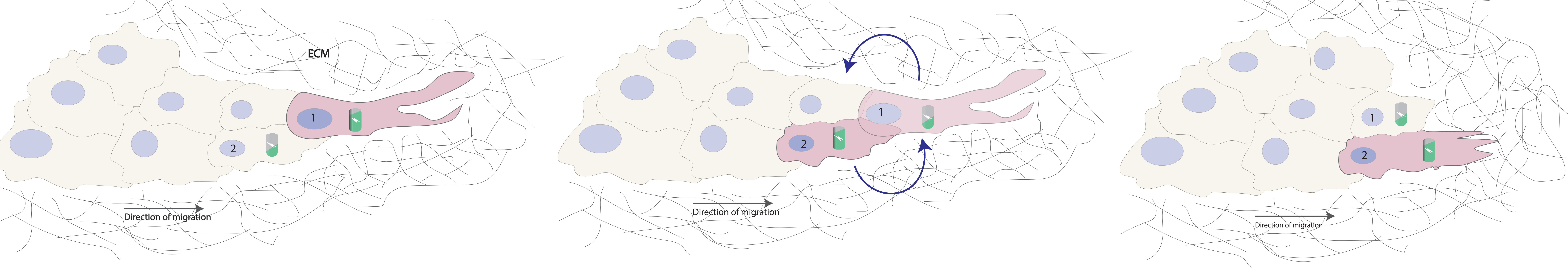




\section{Figure 3}

\section{Single cell migration}
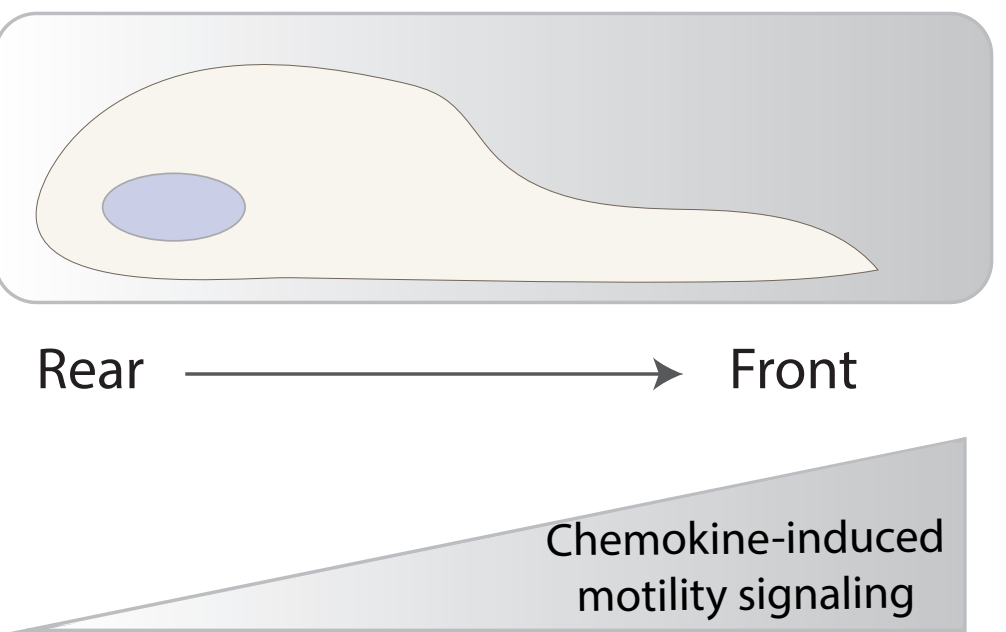

Cdc42/Rac

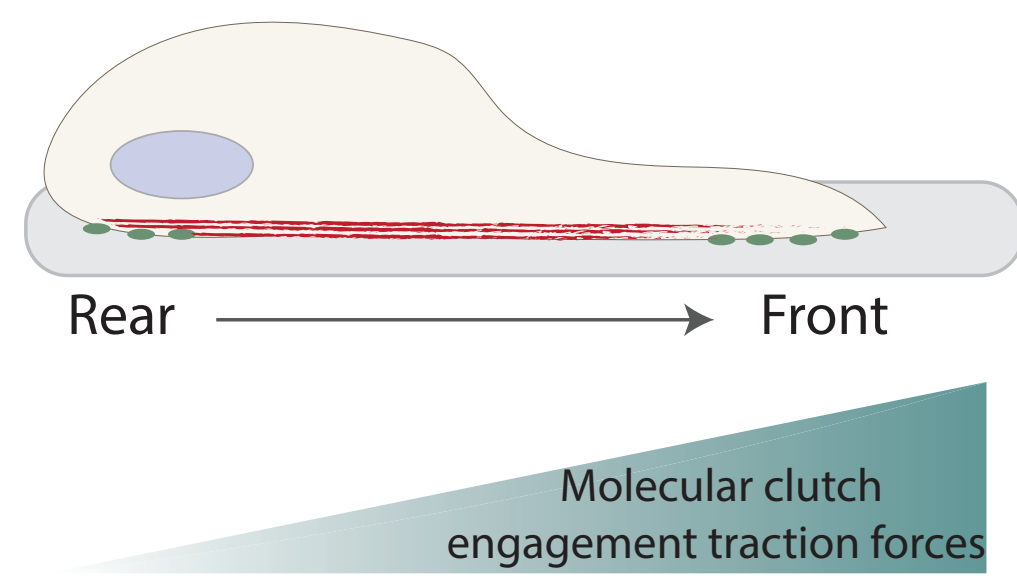

\section{Collective cell migration}

\section{Chemotaxis}

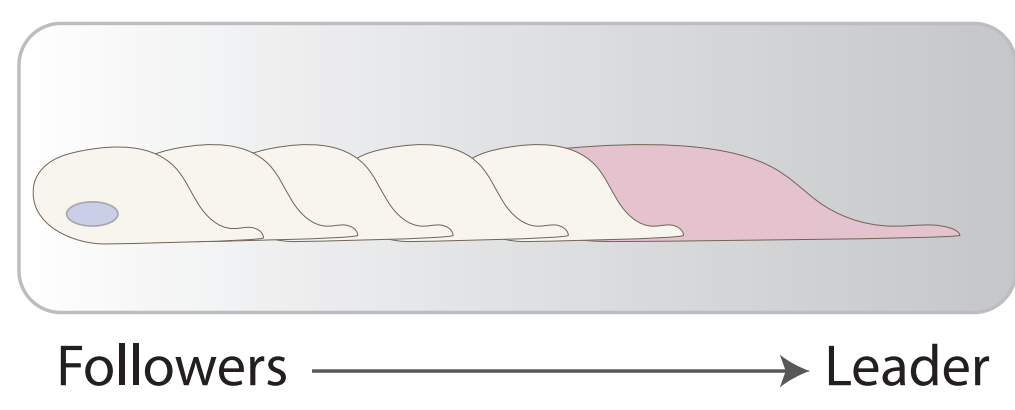

Chemokines degradation

or sequestring

Chemokine-induced motility signaling

\section{Cdc42/Rac}

\section{Durotaxis}

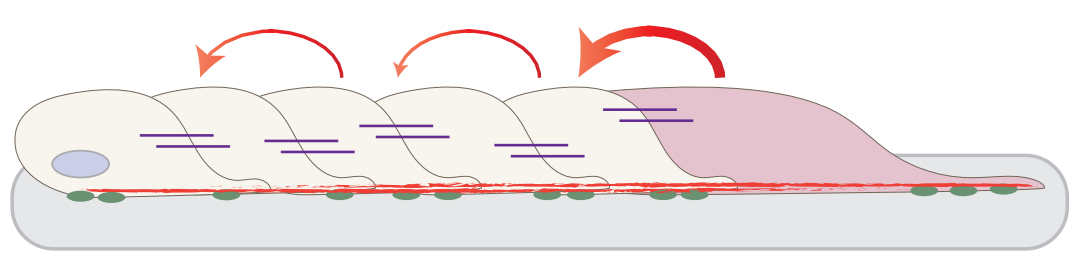

Followers Leader

Molecular clutch engagement traction forces

Force transmission front to rear through AJs 